\title{
Chiral symmetry selection in soft monolayers under vortical flow
}

\author{
Núria Petit-Garrido, ${ }^{[\mathrm{a}]}$ Josep Claret, ${ }^{*[\mathrm{a}]}$ Jordi Ignés-Mullol, ${ }^{[\mathrm{a}]}$ Joan Anton Farrera, ${ }^{[\mathrm{b}]}$ and Francesc \\ Sagués, ${ }^{[\mathrm{a}]}$
}

\begin{abstract}
We report and interpret a new phenomenon of chiral selection in the orientational ordering of soft assembled domains arising spontaneously in Langmuir monolayers of an azobenzene derivative spread at the air-water interface. The orientational chirality of isolated submillimeter domains is unambiguously assessed by means of optical microscopy. The selection process, quantified by means of an enantiomeric excess parameter, is controlled by the stirring of the aqueous subphase. We study the dependence of this process on stirring rate and handedness,
\end{abstract}

stirring time, temperature, and initial state of the monolayer. We discuss the influence of the concomitant cis-trans isomerisation on the reported chiral selection process.

\begin{abstract}
Catalan: En aquest article presentem i interpretem un nou fenomen de selecció quiral de l'ordre orientacional en monocapes de Langmuir de derivats azobenzènics mitjançant l'agitació (vòrtex) de la subfase que suporta la monocapa. A partir de l'observació de les estructures amb microscòpia òtica podem quantificar l'excés enantiomeric sense cap ambigüitat.
\end{abstract}

Estudiem l'efecte dels diferents paràmetres de control sobre aquest fenomen i discutim la influència de la isomerització cis-trans sobre el procés de selecció quiral.

Keywords: chiral selection $•$ Langmuir monolayers - stirring effects -azobenzenes $\bullet$ mesophases

\section{Introduction}

Control of chirality at different hierarchical levels, from complex molecular architectures to crystal structures and soft assembled materials, is a fundamental issue in basic and applied chemistry [1, 2]. Particularly evocated has been the idea to use the chiral force associated to fluid whirling, i.e. hydrodynamic vortices [3, 4], to promote chirality. In this respect, the possibility to break the chiral symmetry of solid materials when crystallized under stirring has been widely recognized since the first observation was reported for supersaturated solutions of sodium chlorate [5-7]. Similar results for

[a] N. Petit-Garrido, Dr. J. Claret, Dr. J. Ignés-Mullol, Prof. Dr. F. Sagués Departament de Química Física

Institut de Nanociència i Nanotecnologia, Universitat de Barcelona Martí i Franqués 1, 08028 Barcelona, Catalunya (Spain) Fax: (+34) 93-402-12-31

E-mail: j.claret@ub.edu

[b] Dr. J. A. Farrera

Department de Química Orgànica

Universitat de Barcelona

Martí i Franqués 1, 08028 Barcelona, Catalunya (Spain) saturated suspensions ground with glass beads have been published more recently referring to the above mentioned inorganic salt [8] and to an amino-acid derivative [9]. When referring to soft materials the question is still more intriguing. Observations that a dominant chirality of self-organized assemblies of achiral molecules could be induced by stirring have appeared during this last decade [10]. An even more striking report published by some of us pointed out to the possibility not only of inducing but to select at will the handedness of labile condensed mesophases by appropriately choosing the stirring vorticity (CW or CCW) [11-13]. The latter phenomenon has been revisited in recent years, extending the discussion to its reversibility or "dynamic nature" [14-18]. However, interpretation of these circular dichroism-based observations has raised some debate due to the need to discern intrinsic chirality of the condensates from instrumental artefacts [19].

Here, a new and robust realization of such vortically-mediated chiral selection in a softly aggregated material is reported. We refer to labile monolayers spread at the air-water interface when moderately sheared by the stirring of the aqueous subphase. Our scenario is different from the previously mentioned situations in several respects. First and more important, chiral selection occurs at the phase boundary of an interfacial system. Indeed, the imposed chiral influence, being a genuine three-dimensional macroscopic force applied to the aqueous bulk phase, is downscale transferred to the two-dimensional, monomolecular thick monolayer residing on top of it. Moreover, our observations use polarized light reflection microscopy that directly reveals the molecular self-assembling order 
inside the aggregates, making any further interpretation of the measurements unnecessary.

The experimental system consists of Langmuir monolayers of an azobenzene derivative, 4-[4-[(4-octylphenyl/azo]-phenoxy] butanoic acid (8Az3COOH on what follows) (Fig. 1). The different nature of its cis and trans isomers on what respects to both their molecular configuration and electric dipolar properties results in distinctive surfactant properties as revealed by polarizing reflection microscopy at the Brewster angle (BAM)[20, 21]. Monolayers of the curved and polar cis-isomer do not exhibit structured mesophases. Conversely, different textures, featuring long-range orientational order [22], are typical of the stretched and apolar trans-form. It is very important to stress that, under room temperature and light conditions, the cis component slowly transforms into the trans isomer, leading to the nucleation of textured elliptical domains. After a slow and complex growth and coalescence processes, the latter transform into circular domains, which evolve into a typical trans monolayer featuring large patches (Smectic-C like) with small reflectivity gradients [23].

The relevant scenario for our purposes is a mixed cis-trans monolayer. After spreading, this particular system displays a twodimensional, emulsion-like biphasic state composed of circular trans-enriched droplets (two-dimensional disks of tens of microns typical diameter) embedded in a cis-rich matrix. BAM images capture the birefringence of these domains, whereas the cis-rich phase appears isotropic. Under the appropriate experimental conditions, the domains are characterized by a continuous change of reflectivity around a central point (Fig. 1). Reflectivity patterns unambiguously reveal [24] that molecules are tangentially organized following the circular contour (in-plane projections of the molecular tails are perpendicular to the radial direction), defining clockwise (CW) or counter-clockwise (CCW) bend-like textures (Fig. 1).

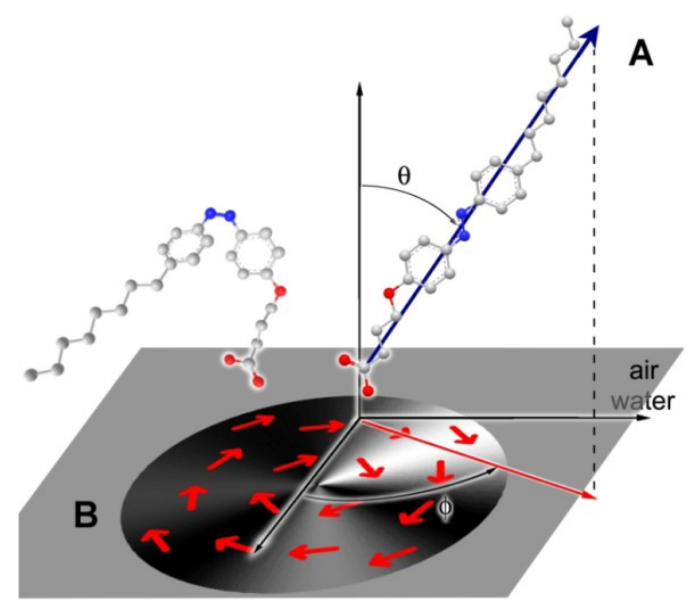

Figure 1. A) Arrangement of the amphiphilic molecules at the air-water interface. In the trans form they organize in labile condensates featuring long-range orientational order, characterized by a uniform molecular tilt, $\theta$, and a spatially varying azimuth, $\phi$. B) The local reflectivity of the monolayer is measured as gray-scale images with Brewster angle microscopy (BAM), and can be unambiguously mapped to spatial distributions of the azimuth (red arrows), which, in our case, is purely rotational (bend-like texture), either $\mathrm{CW}$, as shown, or CCW.

In the absence of any chiral influence, the monolayer appears "racemic", i.e., with (statistically) equilibrated amounts of CW and CCW domains (Fig. 2.A). This is consistent with the achiral nature of the original molecule. The anticipated conclusion of this report is that the orientational chirality of these mesoscopic (sub-millimeter) circular trans-rich domains of the achiral amphipile $8 \mathrm{Az} 3 \mathrm{COOH}$ is decided by the handedness of an applied underneath vortical flow.

\section{Results and Discussion}

The experimental protocol leading to the chiral selection involves two successive temporal stages: the spreading time, $t_{s p}$, from monolayer spreading until starting to stir the water subphase; and the stirring time, $t_{s t}$. The monolayer is maintained at room temperature and light conditions at all times.

Figure 2 shows a mixed cis-trans $8 \mathrm{Az} 3 \mathrm{COOH}$ monolayer prepared at rest (A), and after CW (B) or CCW (C) stirring for $t_{s p}=$ 0 , i.e., spreading on a stirred subphase, and for $t_{s t}=2$ minutes, which is the stirring time that maximizes the chiral selection (see below). Images clearly show the prevalence of $\mathrm{CW}$ (resp. $\mathrm{CCW}$ ) domains formed under $\mathrm{CW}$ (resp. CCW) vorticity and a statistically equilibrated amount of both orientations in the absence of the chiral influence.
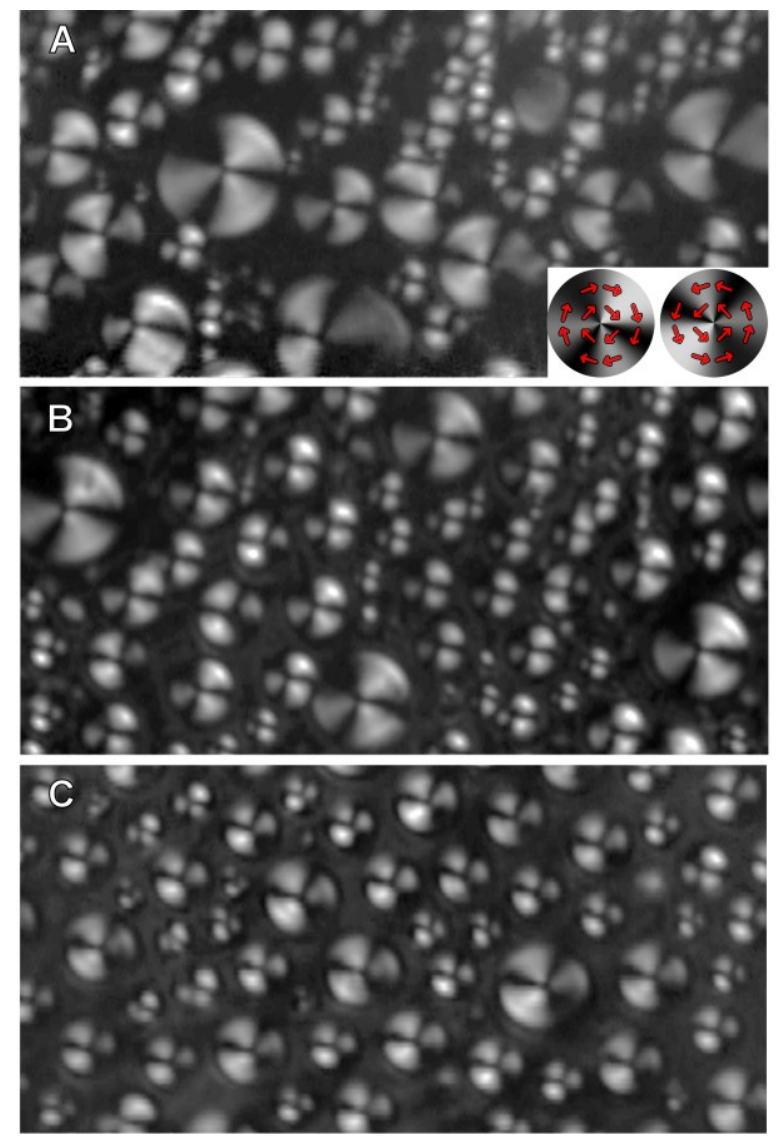

Figure 2. Typical images of mixed cis/trans-8Az3COOH monolayers at low surface pressure prepared without stirring (A), with $\Omega=1000 \mathrm{rpm}$ of CW stirring (B), and with $\Omega=1000 \mathrm{rpm}$ of CCW stirring (C) for $t_{s t}=2$ minutes and $t_{s p}=0$ (see text). A sketch with two domains of opposite chirality is shown in panel A. Notice the different orientation of the three dark extinction brushes to easily identify each handedness. The width of the images is $546 \mu \mathrm{m}$.

Dominance of a particular chirality is measured in terms of the enantiomeric excess of domains with a particular sign (here we choose $\mathrm{CW}$ ),

$$
e_{C W}=\frac{2 n_{C W}-n_{T}}{n_{T}} 100,
$$


where $n_{C W}$ stands for the number of $\mathrm{CW}$ domains with respect to the total number $n_{T}$. In a typical experiment $n_{T}$ is several hundreds.

On what follows, we will discuss the effect of different parameters on this chiral symmetry selection process in order to achieve a comprehensive understanding of the phenomenon.

Role of stirring rate: A systematic series of experiments for different stirring rates has been performed and the results are summarized in Fig. 3. Following the just mentioned experimental protocol $\left(t_{s p}=0, t_{s t}=2\right.$ minutes), the enantiomeric excess is hardly detectable below $\Omega=500 \mathrm{rpm}$ and saturates at a value close to $50 \%$ for $\Omega=1000 \mathrm{rpm}$, i.e., the number of domains with the same handedness as the vortical stirring is three times larger than those with the opposite orientation. When plotted in terms of the applied stirring rate, the enantiomeric excess describes a pitchfork-like bifurcation.

We have performed particle image velocimetry (PIV) measurements of surface flows that confirm that linear speed steadily increases with the stirring rate within the entire range of investigated values. We thus conclude that the upper bound in the extent of chiral selection has to be considered as an intrinsic property of the process.

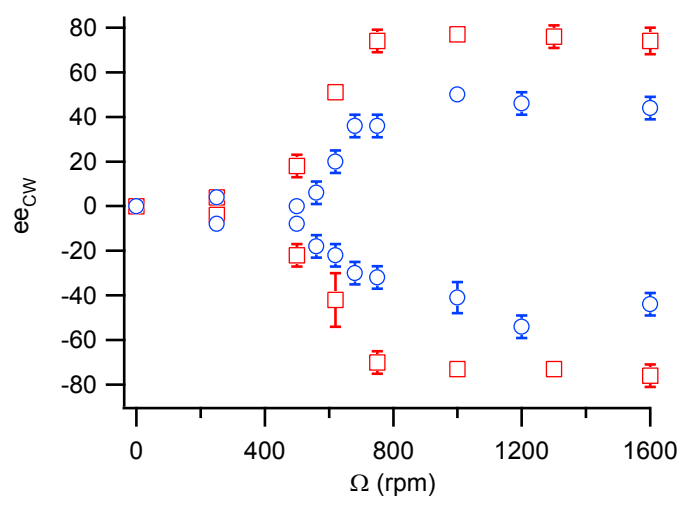

Figure 3. Enatiomeric excess plotted in terms of the applied stirring rate for an initial cis-trans monolayer (circles), and for an initial cis-rich monolayer (squares).

Role of initial monolayer composition: The initial state of the monolayer has significant quantitative effects on the process of chiral selection by stirring. This is evidenced when we consider an alternative protocol by preparing the azobenzene-based monolayer in the single-phase cis-richform, allowing spontaneous nucleation of trans domains to take place under subphase stirring. Results are jointly presented for the sake of comparison in Fig. 3. Data are obtained using $t_{s p}=0$ and $t_{s t}=20$ minutes, the experimental conditions that maximize the selection process under this protocol (see below). The existence of a threshold stirring rate is not so obvious in this case. What these data put into evidence is that chiral selection is more efficient under these conditions at all stirring rates. Although perfect discrimination is not reached, the saturation value is significantly larger for the initially cis-rich monolayer.

The essential interplay between cis and trans isomers in the reported chiral selection is further confirmed by experiments conducted after spreading a pure trans monolayer. In this case, chiral selection is totally absent, and no signature of enantiomeric excess is detected within the entire range of investigated stirring conditions.

Role of temperature: Observations indicate that chiral selection is less effective when increasing temperature by a few degrees above room temperature. In fact, this process does not take place for temperatures higher than $35^{\circ} \mathrm{C}$ in $8 \mathrm{Az} 3 \mathrm{COOH}$ Langmuir monolayers

Mechanism of the chiral symmetry breaking process: The reported observations admit a precise interpretation in terms of a mechanism that explains both the chiral-symmetry breaking and the process of chiral selection itself. The experimental results reported above strongly suggest that handedness selection is promoted by the presence of the cis isomer in the monolayer. Thus, a comprehensive picture of the phenomenon at hand can be obtained after carefully monitoring the slow coarsening dynamics of a cis-rich monolayer kept at rest under the same surface pressure, temperature, and light conditions as for the experiments under stirring.

BAM observations of the temporal evolution of an initially cisrich monolayer under room light reveal the progressive nucleation of trans domains as a consequence of the slow cis $\rightarrow$ trans isomerisation. The emerging condensation nuclei promote further cis $\rightarrow$ trans isomerisation by adopting an elliptical, rather than circular, shape, complying in this way with the tendency of the azobenzene groups in the stretched trans component to assemble into face-to-face H-aggregates [24, 29]. As a matter of fact, ellipsoidal, spindle-like, droplets (tactoids) have also been reported during the isotropic-nematic first order phase transitionss [25-27] of different thermotropic liquid crystals.

Analysis of BAM images show that trans molecules are tilted along the long axis of the ellipse and are tangential to the domain boundary (Fig. 4). This configuration results in the formation of two singularities or defects of the orientational field at the ellipse poles. Moreover, the head/tail asymmetry inherent to any amphiphile at the air-water interface makes the nature of these two defects at the equatorial poles different: one defect is strictly pinched at the domain boundary whereas the second one escapes towards the remaining cis-enriched surrounding pool.
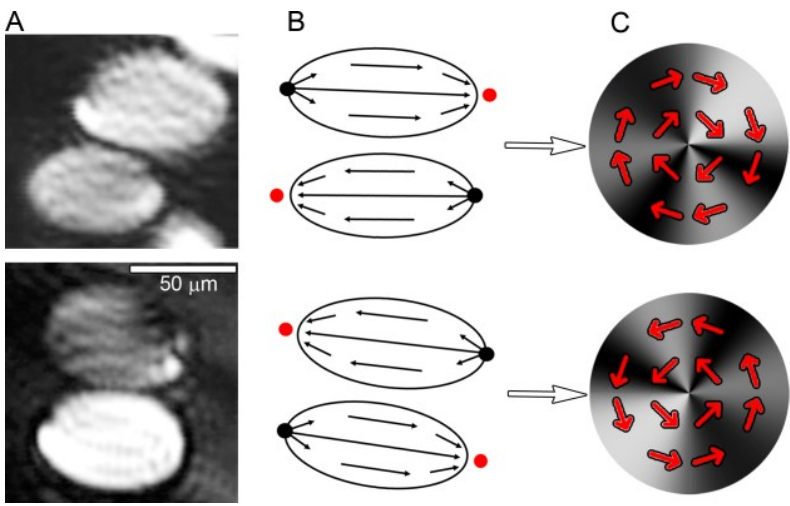

Figure 4. Sketch of the coalescence of two elliptical domains leading to the formation of two enantiomorphic CW (top) and CCW (bottom) bend circular domains (C). The local in-plane orientation of amphiphilic molecules corresponding to BAM images in (A) is sketched in (B). Black and red dots denote the two asymmetric equatorial defects at the poles of the ellipses. 
We have observed the formation of circular bend-like chiral domains through the coalescence of pairs of ellipses with antiparallel molecular orientation, after a delicate process of defect creation and annihilation [30]. The chirality of the resulting domains in turn reflects the relative orientation of their parent ellipses, which constitute enantiomorphic configurations of oppositely directed dipoles (Fig. 4). The distinctive effect of stirring is attributed to the shear forces transmitted from the swirling subphase that, depending on the signed vorticity, would kinetically favour fusion of a particular enantiomeric arrangement of the two ellipses over the opposite configuration [30].
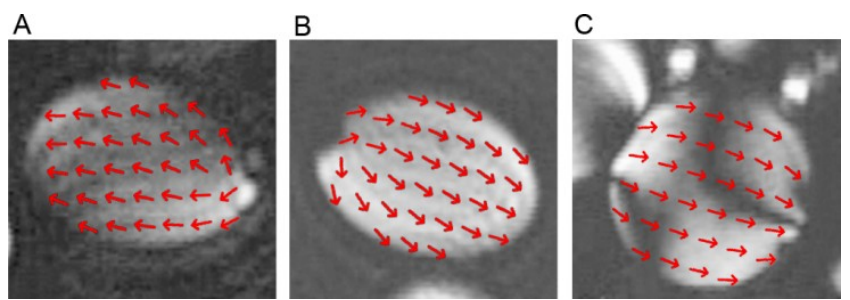

Figure 5. The two antiparallel configurations of elliptical trans domains formed at room temperature $(\mathrm{A}, \mathrm{B})$, and the symmetric double-boojum trans domain observed above $30^{\circ} \mathrm{C}(\mathrm{C})$. The estimated in-plane orientation of the amphiphilic molecules is overlaid on the BAM images.

The essential role of elliptical domains in the reported chiral selection mechanism is supported by the fact that $e e_{C W}$ vanishes at temperatures over $35^{\circ} \mathrm{C}$, regardless of the initial monolayer composition and experimental protocol. At these temperatures, elliptical domains of the trans aggregated material are not stable and transform into circular ones, displaying a double-boojum configuration with two defects pinched at opposite poles (Fig. 5). This is strong indirect evidence that the presence of ellipses with long-axis bipolar asymmetry is the key ingredient for chiral discrimination in these soft monolayers.

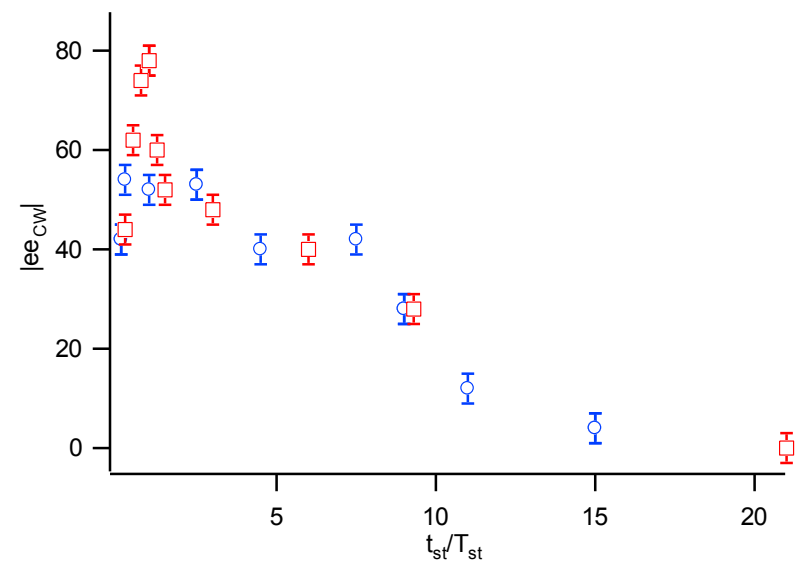

Figure 6. Dependence of $\left|e e_{C W}\right|$ on stirring time at $\Omega=1000 \mathrm{rpm}$ for a mixed cis-trans (circles) and for a cis-rich monolayer (squares) of $8 \mathrm{Az} 3 \mathrm{COOH}$. The time axis has been rescaled with the corresponding optimal stirring time, $T_{s t}$ (see text).

Stirring period and stirring cycle: Concerning the stirring time, $t_{s t}$, the general observation is a steady increase of the absolute value of $e e_{C W}$ with $t_{s t}$ up to an optimal value, $T_{s t}$, which depends on the initial state of the monolayer, and a subsequent steady decrease for larger times. Based on these observations, data reported in Fig. 3 are obtained with $t_{s t}=2$ min for a mixed cis-trans monolayer, and $t_{s t}=$ $20 \mathrm{~min}$ for a $c i s-$ rich monolayer. Data for the two initial conditions are presented in Fig. 6 by plotting $\left|e e_{C W}\right|$ vs. $t_{s t} / T_{s t}$, the rescaled stirring time. The significant overlap between the two data series reveals a common trend in the effect of stirring time. Enantiomeric excesses can also be effectively induced when a monolayer is stirred a time after it has been spread over a quiescent subphase (we have tested $t_{s p}$ in the range $2-30$ minutes), although $e e_{C W}$ is nearly halved with respect to the optimal protocol $t_{s p}=0$.

Table 1. Values of $e e_{C W}$ after different stirring cycles combining $\mathrm{CW}$ and $\mathrm{CCW}$ handedness at $\Omega=1000 \mathrm{rpm}$.

\begin{tabular}{cccc}
\hline Stirring sense & $\begin{array}{c}\text { Stirring time } \\
(\mathrm{min})\end{array}$ & $\% \mathrm{CW}$ & $\mathrm{ee}_{\mathrm{CW}}$ \\
\hline $\mathrm{CW} \rightarrow \mathrm{CCW}$ & $\begin{array}{r}\mathrm{t}_{1, \mathrm{CW}}=2 \\
\mathrm{t}_{2, \mathrm{CCW}}=2 \\
\mathrm{t}\end{array}$ & 34 & -32 \\
$\mathrm{t}, \mathrm{CCW}=2$ & 67 & 34 \\
$\mathrm{t} \mathrm{t}_{2, \mathrm{CW}}=2$ & & \\
$\mathrm{CW} \rightarrow \mathrm{CCW}$ & $\mathrm{t}_{1, \mathrm{CW}}=2$ & 39 & -22 \\
& $\mathrm{t}_{2, \mathrm{CCW}}=8$ & & 30 \\
$\mathrm{CCW} \rightarrow \mathrm{CW}$ & $\mathrm{t}_{1, \mathrm{CCW}}=2$ & 65 & -24 \\
& $\mathrm{t}_{2, \mathrm{CW}}=8$ & & \\
$\mathrm{CW} \rightarrow \mathrm{CCW}$ & $\mathrm{t}_{1, \mathrm{CW}}=8$ & 38 & 37 \\
& $\mathrm{t}_{2, \mathrm{CCW}}=2$ & & \\
$\mathrm{CCW} \rightarrow \mathrm{CW}$ & $\mathrm{t}_{1, \mathrm{CCW}}=8$ & 63 & \\
\hline $\mathrm{t}_{2, \mathrm{CW}}=2$ & & & \\
\hline
\end{tabular}

We propose that the steady reduction in $e e_{C W}$ at long stirring times is the result of shear grinding of the existing bend-like trans domains, which in the end leads to monolayers featuring a racemic mixture of $\mathrm{CW}$ and $\mathrm{CCW}$ domains. In fact, the same mechanism might enhance the growth of incipient elliptical domains in the presence of spontaneous cis-trans isomerisation. This could explain why $e e_{C W}$ grows much more slowly with the stirring time when the initial condition is poor in the trans enantiomer (cis-rich monolayer) As the room-light photo-stationary composition is spontaneously approached, cis $\rightarrow$ trans isomerisation in the spread monolayer becomes negligible, and disgreggated material now self-assembles in bend-like domains with random chirality. The enantiomeric excess also vanishes when a stirred monolayer relaxes at rest. Besides nucleation of bend-like domains of random chirality, spontaneous fusion events between different types of domains [24] significantly contribute to this degradation. Accordingly, we limit data recording to the first 30 minutes after stirring is stopped.

The proposed grinding mechanism helps to explain the fact that chiral selection is also effective even when stirring is applied on a monolayer spread at rest $\left(t_{s p}>0\right)$, or the reversibility in the enantiomeric excess upon inversion of the stirring handedness. Indeed, we have designed experiments where the stirring cycle is split into two steps, of either equal or different duration, having opposite vorticity. Independently of the detailed prescription, results indicate that the observed chiral dominance always corresponds to the vorticity of the later applied stirring, although obtained enantiomeric excesses are smaller than those found when keeping stirring unchanged for similar total times (Table 1). 


\section{Conclusion}

In summary, we have reported and interpreted a new phenomenon of chiral selection in the orientational ordering arising in Langmuir monolayers of an azobenzene derivative at the air-water interface. In particular, we focus on isolated mesophase domains, which arise spontaneously upon coexistence of the cis and trans enantiomers of the surfactant. The orientational chirality of these domains is unambiguously observed by means of optical microscopy, is controlled by the stirring of the aqueous subphase, and is quantified by means of an enantiomeric excess parameter. We have reported a comprehensive study of control parameters that have an influence on the chiral selection process, such as the subphase stirring rate and sign, stirring time, temperature, and initial state of the monolayer. This process is influenced by the spontaneous cis-trans isomerisation of the compound, and we conclude that the optimum selection (enantiomeric excess up to $90 \%$ ) is achieved when the presence of cis isomer is maximized in initial composition of the monolayer. Although the reported observations refer to a particular azobenzene derivative, we can envisage other scenarios involving different surfactants.

Our results reinforce the role of interfacial flows of primordial achiral components on the origin of homochiral terrestrial life [3133 ], based on their ability to mould templates to potentially elicit a particular handedness in biopolymers [34]. We also anticipate possibilities for enantioselective catalysis, either directly on aqueous interfaces [35], or by further transfer of the monolayer to a solid support [36]. Concerning this point, we have successfully transferred these domains by the Langmuir-Blodgett method on a glass slide, with the aim of building liquid crystal cells with patterns of a selected chirality preference. Preliminary results show the ability of these templates to orient thin liquid crystal layers [37].

\section{Experimental Section}

In our experiments, c.a. $40 \mathrm{ml}$ of pure water (Millipore Milli-Q quality) is poured in a Petri dish ( $8 \mathrm{~cm}$ diameter, $2.5 \mathrm{~cm}$ height) and is stirred at a constant rate $\Omega,(0<\Omega<$ $1600 \mathrm{rpm}$ ) by means of a Reversible Magnetic Stirrer (J.P. Selecta, Spain) with a submerged cylindrical Teflon-coated magnetic bar $(8 \mathrm{~mm}$ long, $3 \mathrm{~mm}$ diameter). Monolayers are prepared by carefully depositing one drop $(2 \mu \mathrm{l})$ of a chloroform (Bake p.a.) solution (c.a. $1 \mathrm{mM}$ ) of custom-synthesized $8 \mathrm{Az} 3 \mathrm{COOH}$ [23]. When the subphase is stirred, the drop of the spreading solution is deposited at the outer part of the vortical funnel. The spreading solution for a mixed cis-trans monolayer is a photostationary mixture with the room light of both isomers. To prepare a cis-rich and a trans-rich monolayer, the spreading solutions are either irradiated with UV light for ten minutes or kept overnight in the dark, respectively. Experiments are conducted following the appropriate protocol described above at room-temperature $\left(24-26^{\circ} \mathrm{C}\right)$ with a surface pressure below $1 \mathrm{mN} \mathrm{m}^{-1}$, unless otherwise stated. These are the optimal conditions that maximize the monolayer lability without degrading the chiral selection process.

After stopping stirring, BAM images of the monolayer are recorded during c.a. 30 min. Recording during stirring is unpractical, due to curvature of the interface and the low sensitivity of high-speed cameras. BAM images were obtained using a custom-built instrument, with the analyzer set at $60^{\circ}$ counterclockwise with respect to the plane of incidence, which contains the $y$-axis of the images. An XY translation stage enables to scan the air/water interface, Image analysis has been performed by means of the software ImageJ [38].

\section{Acknowledgements}

We acknowledge financial support by MICINN (Projects FIS2006-03525 and FIS2010-21924-C02-01) and by DURSI (Project 2009 SGR 1055). N. P. acknowledges support from MICINN (Grant AP2007-01103). We are grateful to Laia Josa-Culleré for her collaboration in the measurements and data analysis corresponding to Figure 6.
[1] J. J. L. M. Cornelissien, A. E. Rowan, R. J. M. Nolte, N. A. J. M. Sommerdijk, Chem. Rev. 2001, 101, 4039

[2] L. Pérez-García, D. Amabilino, Chem. Soc. Rev. 2007, 36, 941

[3] G. H. Wagnière, On Chirality and the Universal Asymmetry, WILEY-VCH, Weinheim, 2007, pp. 128-131

[4] A. Guijarro, M. Yus, The Origin of chirality in the molecules of life : a revision from awareness to the current theories and perspectives of this unsolved problem, Royal Society of Chemistry, Cambridge, 2009

[5] D. P. Kondepudi, R. J. Kaufman, N. Singh, Science 1990, 250, 975

[6] J. M. McBride, R. L. Carter, Angew. Chem. Int. Ed. 1991, 30,293

[7] M. Uwaha, J. Phys. Soc. Jpn. 2004, 73, 2601

[8] C. Viedma, Phys. Rev. Lett. 2005, 94, 065504

[9] W. L. Noorduin, T. Izumi, A. Millemaggi, M. Leeman, H. Meekes, W. J. P. Van Enckevort, R. M. Kellogg, B. Kaptein, E. Vlieg, D. G. Blackmond, J. Am. Chem. Soc. 2008, 130, 1158

[10] W. Dzwolak, A. Loksztejn, A. Galinska-Rakoczy, R. Adachi, Y. Goto, L. Rupnicki, J. Am. Chem. Soc. 2007, 129, 7517

[11] J. M. Ribó, J. Crusats, F. Sagués, J. Claret, R. Rubires, Science 2001, 292, 2063

[12] J. Crusats, J. Claret, I. Díez-Pérez, Z. El-Hachemi, H. Garcia-Ortega, R Rubires, F. Sagués, J. M. Ribó, Chem. Comm. 2003, 1588

[13] O. Ohno, Y. Kaizu, H. Kobayashi, J. Chem. Phys. 1993, 99, 4128

[14] T. Yamaguchi, T. Kimura, H. Matsuda, T. Aida, Angew. Chem. Int. Ed. 2004, 43,6350

[15] A. Tsuda, M. A. Alam, T. Harada, T. Yamaguchi, N. Ishii, T. Aida, Angew. Chem., Int. Ed. 2007, 46, 8198

[16] A. D'Urso, R. Randazzo, L. Lo Faro, R. Purrello, Angew. Chem. Int. Ed. 2010, 49, 108

[17] O. Arteaga, A. Canillas, R. Purrello, J. M. Ribó, Opt. Lett. 2009, 34, 2177

[18] M. Wolffs, S. J. George, Z. Tomovic, S. C. J. Meskers, A. Schenning, E. W Meijer, Angew. Chem. Int. Ed. 2007, 46, 8203

[19] G. P. Spada, Angew. Chem. Int. Ed. 2008, 47, 636

[20] S. Hénon, J. Meunier, Rev. Sci. Instrum. 1991, 62, 936

[21] D. Hönig, D. Möbius, J. Phys. Chem. 1991, 95, 4590

[22] T. M. Fischer, R. F. Bruinsma, C. M. Knobler, Phys. Rev. E 1994, 50, 413

[23] J. Crusats, R. Albalat, J. Claret, J. Ignés-Mullol, F. Sagués, Langmuir 2004, 20, 8668

[24] J. Ignés-Mullol, J. Claret, R. Albalat, J. Crusats, R. Reigada, M. T. MartínRomero, F. Sagues, Langmuir 2005, 21, 2948

[25] Y. A. Nastishin, H. Liu, T. Schneider, V. Nazarenko, R. Vasyuta, S. V. Shiyanovskii, O. D. Lavrentovich, Phys. Rev. E 2005, 72, 041711

[26] J. Viamontes, P. W. Oakes, J. X. Tang, Phys. Rev. Lett. 2006, 97, 118103

[27] L. Tortora, O. D. Lavrentovich, PNAS 2011, 108, 5163

[28] N. M. Silvestre, P. Patricio, M. M. Telo da Gama, Phys. Rev. E 2006, 74, 021706

[29] J. M. Pedrosa, M. T. Martín-Romero, L. Camacho, D. Möbius, J. Phys. Chem. B 2002, 106, 2583

[30] N. Petit-Garrido, J. Ignés-Mullol, J. Claret, F. Sagués, Phys. Rev. Lett. 2009, 103,237802

[31] L. D. Barron, Space Sci. Rev. 2008, 135, 187

[32] R. Plasson, D. K. Kondepudi, H. Bersini, A. Commeyras, K. Asakura, Chirality 2007, 19, 589

[33] I. Weissbuch, M. Lahav, Chem. Rev. 2011, 111, 3236

[34] R. M. Hazen, Am. Mineral. 2006, 91, 1715

[35] H. Zepik, E. Shavit, M. Tang, T. R. Jensen, K. Kjaer, G. Bolbach, L. Leiserowitz, I. Weissbuch, M. Lahav, Science 2002, 295, 1266 
[36] K. Katsonis, H. Xu, R. M. Haak, T. Kudernac, Z. Tomovic, S. George, M. V. de Auweraer, A. P. H. J. Schenning, E. W. Meijer, B. L. Feringa, Angew. Chem. Int. Ed. 2008, 47, 4997

[37] N. Petit-Garrido, R. P. Trivedi, J. Ignés-Mullol, J. Claret, C. Lapointe, F Sagués, I. I. Smalyukh, Phys. Rev. Lett. 2011, 107, 177801.

Received: ((will be filled in by the editorial staff)) Revised: ((will be filled in by the editorial staff))

[38] W. S. Rasband, ImageJ 2011, http://rsb.info.nih.gov/ij/ Published online: ((will be filled in by the editorial staff)) 


\section{Entry for the Table of Contents}

Chiral Selection

Núria Petit-Garrido, Josep Claret, Jordi Ignés-Mullol, Joan Anton Farrera, and Francesc Sagués* Page - Page

Chiral symmetry selection in soft monolayers under vortical flow

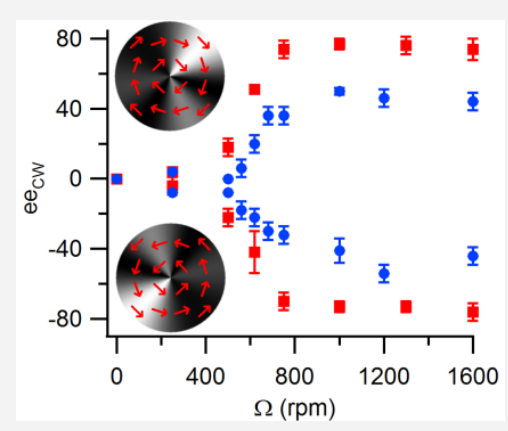

A swirl of surfactants. The orientational chirality of submillimetre domains arising spontaneously in Langmuir monolayers of achiral surfactants is selected by means of a vortical flow under the surface. The enantiomeric excess is directly assessed by means of polarizing optical microscopy, and depends on forcing conditions and initial monolayer state. 